\title{
Modelling Decision-Making Processes in the Management Support of the Manufacturing Element in the Logistic Supply Chain
}

\author{
Robert Bucki ${ }^{1}$ and Petr Suchánek ${ }^{2}$ \\ ${ }^{1}$ Institute of Management and Information Technology, Bielsko-Biala, Poland \\ ${ }^{2}$ Silesian University in Opava, School of Business Administration in Karviná, Karviná, Czech Republic
}

Correspondence should be addressed to Petr Suchánek; suchanek@opf.slu.cz

Received 23 February 2017; Revised 25 April 2017; Accepted 9 May 2017; Published 28 June 2017

Academic Editor: Vladimir Modrak

Copyright (C) 2017 Robert Bucki and Petr Suchánek. This is an open access article distributed under the Creative Commons Attribution License, which permits unrestricted use, distribution, and reproduction in any medium, provided the original work is properly cited.

\begin{abstract}
This paper highlights the problems of mathematical modelling for a specific element of the logistic supply chain, that is, the manufacturing system. The complex manufacturing system consisting of a determined number of parallel subsystems is modelled. The fact that the same manufacturing procedure can be carried out in various locations is emphasised. Control algorithms as well as manufacturing strategies are explained. The equations of state are introduced. The two-stage criterion lets us use the result data generated by the simulator of the production system as the initial data for further processing; however, the main goal remains to minimise the time of the course of production. The precisely elaborated case study implements initial data obtained by preceding simulation procedures carried out in manufacturing systems consisting of three, four, and five subsystems.
\end{abstract}

\section{Introduction}

Nowadays, design and optimisation of logistic and production systems is a demanding and important area. Businesses are pushed to improve their performance, decrease production prices, and increase variability of their products or shorten delivery times. If a company fails to adapt to these requirements, it cannot normally survive in the contemporary market environment. To survive in the highly competitive global economy, manufacturing systems must be able to adapt to new circumstances [1]. Production systems may vary in terms of their complexity. Moreover, many current production companies have geographically separate production sites and factories are often located in different countries in which there are different labour costs which results in a different price per unit time of production. Moving production from one plant to another may be one way to reduce costs, that is, to increase profits. Manufacturing costs seem to be the most important factor but there are also others which managers must take into account. One of them is stability of the region where the manufacturing plant is located. The next one worth considering is reliability of the supply chain. It is also necessary to consider transport availability, distance from customers, and so on. As it is clear from the above and other contexts, a number of various indicators must be taken into account for complexity assessment of selected general process structures while designing the structure or optimising manufacturing tasks [2]. The structure of production processes also depends on the production needs of specific products that may show high variability. Organising production processes is closely related to the process maps and procedures responsible for manufacturing products from individual components [3]. A key prerequisite for the effectiveness of the above and other production systems is the precise definition of the interaction between the machining process and the machine tools [4]. Also, requirements grow for monitoring possible disruptions of production systems [5]. Additionally, parts and quick recovery of the production process in case of faults and other risks linked to the general input-output model which is a production system are to be considered [6]. Manufacturing of the product generally occurs as a series of individual actions that are performed 
manually, mechanically, or a combination of these. Optimisation requires continuously processed orders on individual projects. It can also be a serial production of one product in which you can easily define a set of key performance indicators and manage and automate production $[7,8]$. Today, the standard starting point for the design and optimisation of manufacturing systems is simulation. Computer simulations allow you to test different variants of production quickly. It is possible to test many consequences of the changes in the production process and choose the most effective way of making orders in a short time [9]. Simulations can be used both before the draft design of the production system and for the purpose of optimising the production system and reengineering of production processes, respectively. In both cases it is necessary to treat simulation results as baseline data and information for designing or redesigning the considered system. In addition to defining the structure of production systems (in production and logistics systems especially of discreet nature, see, e.g., [10-12]) simulations are useful for planning production and its sustainability and continuity [13, 14]. Specifically, the simulation can help coordinate the needs of various departments and discovery and management bottlenecks and improve resource allocation, distribution of production between production lines or plants, testing strategies, performance measurement, and so on $[15,16]$. Everything mentioned may result in lower production costs and increase efficiency ultimately. The basis for the simulation is mathematical models. Creating simulation mathematical models allows us to use a variety of methods and approaches. This could include a multiagents approach [17, 18], Petri nets [19], object-oriented approach [20], or many others. For the purposes of logistics and production systems, which are the main focus of this article, heuristic approaches can be effectively used. Additionally, it is possible to define a mathematical simulation model in the selected set of cases. Such a model allows us to obtain information for particular specific optimisation solutions (see, e.g., [21-23] or [24]). If there is a set of production lines, factories, and so on, it is also possible to use some of the theoretical background in the field of modular systems. The goal of every company is to make a profit and search for new markets and, at the same time, look for ways of minimising manufacturing costs. One of them is locating production in certain destinations where labour costs are considerably lower. Nevertheless, manufacturing capacities of companies can be limited to make products in time which results in placing production load in a more competitive location. Customers usually do not need all ready orders at once. Products they require are to be delivered to their logistic centers subsequently, for example, for further processing.

Production systems have various structures; however, they are defined by certain common features. One of them is the tool replacement problem. Tools are replaced either individually or within replacement of the so-called tool stand. Usually, there is a tendency to replace only fully worn out tools. A problem which occurs in the discussed system is the need to replace the stand with tools even if the tools in it are not fully worn out. So far manufacturing problems of this type have been solved on condition that either fully worn out tools or the unnecessary ones are subject to replacement $[25,26]$.

The main goal of this article is to define and simulate the mathematical model for the evaluation of the distribution of production in several plants within one complex company to achieve maximum production in the shortest possible time and then minimise costs of manufacturing. Individual companies may be geographically located in different countries with different labour costs and a production time unit. Individual companies are considered as autonomous systems with the same production possibilities. The model takes into account three variants consisting of 3,4 , or 5 companies which can make orders according to vacancies. To compare all possible variants, the mathematical methods of permutations without repetition are used. The basic criteria for the comparison of efficiencies of the production system as a whole are the time and cost of manufacturing time unit.

Complexity has always been a part of each manufacturing environment. Therefore, there has always been a need to classify them as complex systems where numerous phenomena take place $[27,28]$. The complexity of the selected system is described by its behaviour. All components interact in multiple ways and follow the rules which result from the specification and model of the system. The system is characterised by its interdependencies which are created step by step in accordance with a software engineering approach. Programming complexity is a measure of the interactions of the various elements of the software created for meeting the article objectives. The mutual interdependencies are regulated by the emerging need to make the order matrix elements at the given stages. The state of the discussed system emerges from a collection of preceding interactions of work stands. The system receives orders at random so it is never known in advance what kind of order customers require. Once the order is set, the whole course of calculations depending on each other should be initiated to deliver the solution to meet the stated criteria. These correlated relationships let us create a differentiated structure that can, as a system, interact with its subsystems which are placed in various locations. The organised aspect of this form of complexity emerges from the need to meet accepted criteria without any operator's guidance at the decision-making level. This duty is taken over by heuristic algorithms. The number of machines does not have to be very large for a particular subsystem to have emergent properties. The system implements specified rules which can be invoked to explain the origin of complexity. Tools in stands which are subject to replacement are an important factor of complexity. The system is highly sensitive to initial conditions. The size of the input influences the level of complexity; that is, the state of the system is a function of the number of order matrix elements. In our case, complexity can be treated as a measure of the total number of correlations in each subsystem whose properties are understood as a state of the system. A complex system is defined by its different attributes and presents problems in both mathematical modelling and subsequent simulation testing. The study of the complex system investigates how relationships between tools in work stands and buffer stores, understood 
as interconnected components, give rise to the collective behaviours of the subsystems in the frame of the whole manufacturing system. The equations from which the model of the complex system is developed derive from information theory and represent organised but unpredictable behaviours of the manufacturing system that can be considered fundamentally complex. Simulation of company processes requires a thorough analytical approach. The method presented in this article is proposed for an automotive industry supplier. The output of the analysed global company is represented by various parts used during a car assembly process. The multinational company has its headquarters in the heart of Europe and, from there, customers' orders are directed to dedicated plants for manufacturing. It is possible to adjust manufacturing procedures so that they can be the same in all plants. For comparison reasons it is assumed that all analysed plants have the same arrangements of machines and tools when required. This enables making any order matrix element in any available plant. It is assumed that quality issues are met in every plant of the system. The problem exists because the price of work differs as each macrologistic area is characterised by its autonomous labour costs. However, it is impossible to locate all production output in the plant which would make orders as cheap as possible as there are time constraints. Knowing the initial state of orders as well as time bounds for ordered products the search for the cheapest manufacturing arrangement is initiated. The approach emphasised in the article is an autonomous one as there are no general methods for optimising manufacturing tasks. The model of the system is significant as it eliminates excessive costs, allowing the operator of the system to distribute the orders to the plants whose configuration increases the profits of the global company by minimising the total manufacturing costs. The article focuses on the information approach to the simulation process of the logistic manufacturing system. This kind of approach results from software engineering which has led to creating the dedicated software to carry out required calculations leading to reaching the satisfactory solution.

The proposed model represents one of the possible solutions for optimising production in case of dislocated companies which are at disposal. It is significant as it is possible to use it for a particular realistic case described by the defined structure and, of course, it can be treated as one of the scientific bases for solving similar examples (e.g., adaptation of the model for other structures, implementation of other decision-making algorithms, and expansion of other evaluation parameters, for example, from the set of key performance indicators (KPI) of the company).

\section{Mathematical Model}

Symbols used for logistic system modelling presented in this section are explained in Symbols shown at the end of the paper. They remain in accordance with the standards of mathematical models used for the description and simulation of logistic, production, trade, and other systems.

It is assumed that manufacturing processes take place in the complex system which consists of $A$ subsystems.
Each $\alpha$ th subsystem, $\alpha=1, \ldots, A$, carries out exactly the same production operations; however, their initial state may differ. Orders can be made in any $\alpha$ th manufacturing plant.

The separate manufacturing plant has a serial form and consists of work stands assembled in sequence. The stands are equipped with tools which are subject to wear throughout the manufacturing process. When a tool in a stand is completely worn out, the manufacturing process is continued with the use of another tool in the same stand as long as either its life or the manufacturing route allows it. It is assumed that tools cannot be replaced separately. When all tools in the given stand are worn out or the manufacturing process cannot be continued through this stand, the discussed stand is subject to replacement. There are buffer stores between the work stands.

The manufacturing plant described above can be multiplied which results in creating exactly the same manufacturing system in various locations. It is assumed that $M$ customer can order $N$ types of products.

Let us assume orders to be made are shown in the matrix of orders:

$$
\begin{aligned}
& Z^{k}=\left[z_{m, n}^{k}\right], \\
& \quad k=1, \ldots, K, m=1, \ldots, M, n=1, \ldots, N,
\end{aligned}
$$

where $z_{m, n}^{k}$ is the $n$th order of the $m$ th customer at the $k$ th stage.

If the $n$th product cannot be made for the $m$ th customer then $z_{m, n}^{k}=-1$; otherwise $z_{m, n}^{k} \geq 0$.

Products are made from various types of charges which leads to introducing the adjustment matrix of charges to products:

$$
\begin{aligned}
& \Omega=\left[\omega_{l /(m, n)}\right], \\
& \quad l=1, \ldots, L, m=1, \ldots, M, n=1, \ldots, N,
\end{aligned}
$$

where $\omega_{l /(m, n)}$ is the adjustment of the $n$th order of the $m$ th customer to the $l$ th charge (the number of the charge element).

The discussed manufacturing system takes the form shown in the matrix of structure:

$$
E=\left[e_{i, j}^{\alpha}\right], \quad \alpha=1, \ldots, A, i=1, \ldots, I, j=1, \ldots, J,
$$

where $e_{i, j}^{\alpha}$ is the $i$ th tool in the $j$ th work stand in the $\alpha$ th manufacturing plant.

The route matrix is introduced:

$$
D=\left[d_{j, n}\right], \quad j=1, \ldots, J, n=1, \ldots, N,
$$


where $d_{j, n}$ is the number of the $i$ th tool in the $j$ th work stand used for making the $n$th type product.

The base life matrix is introduced:

$$
G=\left[g_{i, j}\right], \quad i=1, \ldots, I, j=1, \ldots, J,
$$

where $g_{i, j}$ is the number of base elements of a product which can be made by the $i$ th tool in the $j$ th work stand before it becomes worn out.

Let us associate the matrix of coefficients with the previous matrix of life:

$$
\begin{aligned}
& \Psi=\left[\psi_{(m, n) /(i, j)}\right], \\
& m=1, \ldots, M, n=1, \ldots, N, i=1, \ldots, I, j=1, \ldots, J,
\end{aligned}
$$

where $\psi_{(m, n) /(i, j)}$ is the coefficient determining how many units of the $n$th type order for the $m$ th customer can be made by the $i$ th tool in the $j$ th tool stand.

At the same time $\psi_{(m, n) /(i, j)} \geq 1$ if the $n$th type product is made by the $i$ th tool in the $j$ th work stand; otherwise $\psi_{(m, n) /(i, j)}=0$.

The base coefficient for the calculation purpose $\psi_{0}=1$.

The life matrix for the $n$th product of the $m$ th customer takes the following form:

$$
\begin{aligned}
G & =\left[g_{(m, n) /(i, j)}\right], \\
m & =1, \ldots, M, n=1, \ldots, N, i=1, \ldots, I, j=1, \ldots, J,
\end{aligned}
$$

where $g_{(m, n) /(i, j)}$ is the number of $n$th products for the $m$ th customer elements which can be made by the $i$ th tool in the $j$ th work stand.

At the same time $g_{(m, n) /(i, j)}=\psi_{(m, n) /(i, j)} \cdot g_{i, j}, m=1, \ldots$, $M, n=1, \ldots, N, i=1, \ldots, I, j=1, \ldots, J$.

The structure vector of base capacity of buffer stores is introduced:

$$
B=\left[b_{j}^{\alpha}\right], \quad \alpha=1, \ldots, A, j=1, \ldots, J-1,
$$

$$
S_{\alpha}^{k}=\left[s(\alpha)_{(m, n) /(i, j)}^{k}\right], \quad \alpha=1, \ldots, A, k=1, \ldots, K, i=1, \ldots, I, j=1, \ldots, J, m=1, \ldots, M, n=1, \ldots, N
$$

where $s(\alpha)_{(m, n) /(i, j)}^{k}$ is the state of the $i$ th tool in the $j$ th stand in the $\alpha$ th manufacturing system in case of making the $n$th product for the $m$ th customer at the $k$ th stage. At the same time $s(\alpha)_{(m, n) /(i, j)}^{k}=\psi_{n /(i, j)} \cdot s(\alpha)_{i, j}^{k}$.

The base flow capacity of the manufacturing system is defined in the base matrix of flow capacity: where $b_{j}^{\alpha}$ is the base capacity of the buffer store placed behind the $j$ th tool stand in the $\alpha$ th manufacturing system. At the same time $b_{j}^{\alpha}>0$ if the buffer is active; otherwise $b_{j}^{\alpha}=-1$.

It is assumed that each $\alpha$ th manufacturing plant has the same active structure of the buffer store system; however, their capacity may differ.

Let us introduce the matrix of coefficients of the buffer stores:

$$
\begin{aligned}
& \Psi_{b}=\left[\begin{array}{c}
\alpha \\
\psi_{j, n}
\end{array}\right], \\
& \quad \alpha=1, \ldots, A, j=1, \ldots, J-1, n=1, \ldots, N,
\end{aligned}
$$

where $\psi_{j, n}^{\alpha}$ is the coefficient of the $j$ th buffer store in case of storing the $n$th type product in the $\alpha$ th manufacturing system.

The state of the buffer store is expressed as follows:

$$
\begin{aligned}
B^{k}=\left[b_{\alpha / j}^{k}\right], & \\
\alpha & =1, \ldots, A, j=1, \ldots, J-1, k=1, \ldots, K,
\end{aligned}
$$

where $b_{\alpha / j}^{k}$ is the state of the $j$ th buffer store in the $\alpha$ th manufacturing plant at the $k$ th stage.

The manufacturing system is always defined in its current state shown in the base matrix of state:

$$
\begin{aligned}
S^{k} & =\left[s(\alpha)_{i, j}^{k}\right], \\
\alpha & =1, \ldots, A, k=1, \ldots, K, \quad i=1, \ldots, I, j=1, \ldots, J,
\end{aligned}
$$

where $s(\alpha)_{i, j}^{k}$ is the base state of the $i$ th tool in the $j$ th stand in the $\alpha$ th manufacturing system at the $k$ th stage.

The state of the system is recalculated and shown in the matrix of state:

$$
\begin{aligned}
P^{k} & =\left[p(\alpha)_{i, j}^{k}\right], \\
\alpha & =1, \ldots, A, k=1, \ldots, K, i=1, \ldots, I, j=1, \ldots, J,
\end{aligned}
$$

where $p(\alpha)_{i, j}^{k}$ is the base capacity of the $i$ th tool in the $j$ th stand in the $\alpha$ th manufacturing system at the $k$ th stage.

The flow capacity of the system is recalculated and shown in the matrix of flow capacity:

$$
P_{\alpha}^{k}=\left[p(\alpha)_{(m, n) /(i, j)}^{k}\right], \quad \alpha=1, \ldots, A, k=1, \ldots, K, i=1, \ldots, I, j=1, \ldots, J, m=1, \ldots, M, n=1, \ldots, N
$$


where $p(\alpha)_{(m, n) /(i, j)}^{k}$ is the flow capacity of the $i$ th tool in the $j$ th stand in the $\alpha$ th manufacturing system in case of making the $n$th product for the $m$ th customer at the $k$ th stage. At the same time $p(\alpha)_{(m, n) /(i, j)}^{k}=\psi_{n /(i, j)} \cdot p(\alpha)_{i, j}^{k}$.

The flow capacity of the $i$ th tool in the $j$ th stand in the $\alpha$ th manufacturing system in case of making the $n$th product for the $m$ th customer at the $k$ th stage is expressed as follows:

$$
\begin{aligned}
p(\alpha)_{(m, n) /(i, j)}^{k}= & \psi_{(m, n) /(i, j)} \cdot g_{i, j}-\psi_{(m, n) /(i, j)} \\
& \cdot s(\alpha)_{(m, n) /(i, j)}^{k} .
\end{aligned}
$$

The matrix of manufacturing times takes the following form:

$$
\begin{aligned}
& T^{\mathrm{pr}}=\left[\tau_{(m, n) /(i, j)}^{\mathrm{pr}}\right], \\
& m=1, \ldots, M, n=1, \ldots, N, i=1, \ldots, I, j=1, \ldots, J,
\end{aligned}
$$

where $\tau_{(m, n) /(i, j)}^{\mathrm{pr}}$ is the manufacturing time of one unit of the $n$th product for the $m$ th customer by the $i$ th tool of the $j$ th work stand.

The vector of replacement times takes the following form:

$$
T^{\mathrm{repl}}=\left[\tau_{j}^{\mathrm{repl}}\right], \quad j=1, \ldots, J,
$$

where $\tau_{j}^{\text {repl }}$ is the replacement time of tools in the $j$ th work stand.

Let us introduce the vector of unit time costs:

$$
C_{\mathrm{UTC}}=\left[c_{\alpha}\right], \quad \alpha=1, \ldots, A,
$$

where $c_{\alpha}$ is the cost of one time unit of operating the $\alpha$ th plant.

2.1. Equations of State. The state of the complex manufacturing system changes after either any decision concerning producing any $n$th product for the $m$ th customer in any $\alpha$ th manufacturing plant or replacing any $j$ th stand in the $\alpha$ th manufacturing plant:

$$
S_{\alpha}^{0} \longrightarrow \cdots \longrightarrow S_{\alpha}^{k} \longrightarrow \cdots \longrightarrow S_{\alpha}^{K} .
$$

The state of the $i$ th tool in the $j$ th stand in case of making the $n$th product for the $m$ th customer changes consequently throughout the manufacturing course:

$$
\begin{aligned}
s(\alpha)_{(m, n) /(i, j)}^{0} & \longrightarrow \cdots \longrightarrow s(\alpha)_{(m, n) /(i, j)}^{k} \longrightarrow \cdots \\
& \longrightarrow s(\alpha)_{(m, n) /(i, j)}^{K},
\end{aligned}
$$

which can be written in the following form:

$$
\begin{aligned}
& s(\alpha)_{(m, n) /(i, j)}^{k} \\
& \quad=\left\{\begin{array}{l}
s(\alpha)_{(m, n) /(i, j)}^{k-1} \\
s(\alpha)_{(m, n) /(i, j)}^{k-1}+x(\alpha)_{(m, n) /(i, j)}^{k}
\end{array}\right.
\end{aligned}
$$

in case of not making the $n$-th product for the $m$-th customer by the $i$-th tool in the $j$-th stand in the $\alpha$-th manufacturing system at the $k$-th stage, otherwise,

where $x(\alpha)_{(m, n) /(i, j)}^{k}$ is the amount of the $n$th product for the $m$ th customer made by the $i$ th tool in the $j$ th stand in the $\alpha$ th manufacturing system at the $k$ th stage.

The state of the base $i$ th tool in case of replacement the $j$ th stand changes as follows:

$$
s(\alpha)_{i, j}^{k}= \begin{cases}s(\alpha)_{i, j}^{k-1} & \text { if the } j \text {-th stand is replaced } \\ 0 & \text { otherwise. }\end{cases}
$$

The order matrix $Z^{k}$ changes after every decision concerning production activity:

$$
Z^{0} \longrightarrow Z^{1} \longrightarrow \cdots \longrightarrow Z^{k} \longrightarrow \cdots \longrightarrow Z^{K}
$$

The order matrix is modified after every decision about production:

$$
\begin{aligned}
& z_{m, n}^{k} \\
& = \begin{cases}z_{m, n}^{k-1}-x_{m, n}^{k} & \text { in case of making the } n \text {-th product for the } \\
z_{m, n}^{k-1} & m \text {-th customer at the } k \text {-th stage, }\end{cases}
\end{aligned}
$$

where $x_{m, n}^{k}$ is the number of units of the $n$th product for the $m$ th customer made at the $k$ th stage.

2.2. Control Algorithms. There is a need to seek satisfactory solutions by making orders in various locations as they generate various manufacturing costs. Comparing costs allows us to determine where the $n$th order should be manufactured to minimise production costs. In order to control the course of manufacturing there is also a need to introduce sample heuristic algorithms. First of all, it is necessary to determine the $\alpha$ th manufacturing plant to place an order in. Secondly, the $n$th product for the $m$ th customer to be made is to be chosen. The flow capacity means how many elements of the order matrix can be passed through the manufacturing plant before production capabilities of the subsystem are used up. First of all, the operator of the system is obliged to choose 
the $\alpha$ th plant, $\alpha=1, \ldots, A$. For example, if the algorithm of the minimal flow capacity of the production plant is set it means that a plant which can make the fewest number of products is required; then the search for the $\alpha$ th plant meeting this condition is initiated. Having detected such a plant, the algorithm of the maximal order is implemented which means that the biggest order to be made through the available route in the $\alpha$ th plant is required. Such an approach is explained in detail in [21, 29].

2.2.1. The Algorithm of the Maximal Flow Capacity of the Production Plant. This algorithm chooses the $\alpha$ th manufacturing plant on condition that it is characterised by the maximal coefficient of flow capacity $\xi_{\alpha}^{k}=\sum_{i=1}^{I} \sum_{j=1}^{J} p(\alpha)_{(m, n) /(i, j)}^{k}$. To determine the $\lambda$ th manufacturing plant where $1 \leq \lambda \leq A$ condition (25) must be met:

$$
\begin{gathered}
\left(q_{\alpha \_ \text {max }}^{k}=\xi_{\lambda}^{k}\right) \Longleftrightarrow \\
{\left[\xi_{\lambda}^{k}=\max _{1 \leq \alpha \leq A} \xi_{\alpha}^{k}\right] .}
\end{gathered}
$$

2.2.2. The Algorithm of the Minimal Flow Capacity of the Production Plant. This algorithm chooses the $\alpha$ th manufacturing plant on condition that it is characterised by the minimal coefficient of flow capacity $\xi_{\alpha}^{k}=\sum_{i=1}^{I} \sum_{j=1}^{J} p(\alpha)_{(m, n) /(i, j)}^{k}$. To determine the $\lambda$ th plant where $1 \leq \lambda \leq A$ condition (26) must be met:

$$
\begin{gathered}
\left(q_{\alpha \_ \text {min }}^{k}=\xi_{\lambda}^{k}\right) \Longleftrightarrow \\
{\left[\xi_{\lambda}^{k}=\min _{1 \leq \alpha \leq A} \xi_{\alpha}^{k}\right] .}
\end{gathered}
$$

2.2.3. The Algorithm of the Maximal Order. This algorithm chooses the order matrix element characterised by the maximal value of $\gamma_{m, n}^{k}$. To produce the order $z_{\mu, \eta}^{k}, 1 \leq \mu \leq M$, $1 \leq \eta \leq N$, the following condition must be met where $\gamma_{m, n}^{k}=z_{m, n}^{k}$ :

$$
\begin{gathered}
\left(q_{z \_ \text {max }}^{k}=z_{\mu, \eta}^{k}\right) \Longleftrightarrow \\
{\left[\gamma_{\mu, \eta}^{k}=\max _{1 \leq m \leq M} \gamma_{m, n}^{k}\right] .}
\end{gathered}
$$

$$
y^{\prime}(\alpha)_{i, j}^{k}= \begin{cases}1 \quad \text { if the } i \text {-th tool in the } j \text {-th stand in the } \alpha \text {-th manufacturing plant } \\ \text { is implemented in the manufacturing process at the } k \text {-th stage, } \\ 0 \quad \text { otherwise. }\end{cases}
$$

Having generated the best solution from the point of view of minimising time, there is a need to minimise manufacturing costs by means of the criterion of minimising
2.2.4. The Algorithm of the Minimal Order. This algorithm chooses the order matrix element characterised by the minimal value $\gamma_{m, n}^{k}$. To produce the order $z_{\mu, \eta}^{k}, 1 \leq \mu \leq M$, $1 \leq \eta \leq N$, the following condition must be met where $\gamma_{m, n}^{k}=z_{m, n}^{k}$ :

$$
\begin{gathered}
\left(q_{z \_ \text {min }}^{k}=z_{\mu, \eta}^{k}\right) \Longleftrightarrow \\
{\left[\gamma_{\mu, \eta}^{k}=\min _{\substack{1 \leq m \leq M \\
1 \leq n \leq N}} \gamma_{m, n}^{k}\right] .}
\end{gathered}
$$

2.3. Manufacturing Strategies. In order to make orders in the $\alpha$ th subsystem, the following strategies are proposed:

(i) The immediate strategy: a new order is introduced into the manufacturing system as soon as such a possibility emerges.

(ii) The delayed strategy: a new order is introduced into the manufacturing system only then when the preceding one leaves the system.

2.4. Criteria. There are a few criteria to be taken into account; however, the most decisive one in terms of manufacturing under time pressure seems to be the minimal manufacturing time criterion so it is chosen for further consideration. As orders are made in various plants there are corresponding aspects of this problem to be analysed. One of them is a different location of each plant which is connected with different manufacturing costs. It is assumed that each manufacturing process is carried out in accordance with the two-stage criteria specified below. First of all, the criterion of minimising the manufacturing time is introduced:

$$
Q_{T}=\sum_{\alpha=1}^{A} \sum_{k=1}^{K} \sum_{i=1}^{I} \sum_{j=1}^{J} y^{\prime}(\alpha)_{i, j}^{k} \cdot \tau_{(m, n) /(i, j)}^{\mathrm{pr}} \longrightarrow \min ,
$$

where

manufacturing costs:

$$
Q_{C}=\sum_{\alpha=1}^{A} c_{\alpha} \longrightarrow \text { min. }
$$




\section{Case Study}

In order to verify correctness of the assumptions presented in the paper hereby it is necessary to carry out the simulation process with the use of sample data. Apart from obtaining results concerning the minimal manufacturing time it is possible to present other associating results for each simulation process, that is, total replacement time of tools in work stands, lost flow capacity of tools due to premature replacement, and remaining capacity of tools in case of completing making the whole order.
The following general assumptions for the case study are taken into account:

(i) All available manufacturing plants are at the same initial state as follows:

$$
\underset{1 \leq \alpha \leq A}{\exists} S_{\alpha}^{0}=S^{0} .
$$

(ii) There is enough charge material for making the whole order matrix elements.

The simulator used for simulating making order receives data drawn at random from a specified range:

$$
\begin{aligned}
& Z^{0}=\left[\begin{array}{lllll}
24 & 32 & 50 & 51 & 29 \\
39 & 24 & 33 & 28 & 33 \\
46 & 47 & 48 & 33 & 39 \\
46 & 43 & 51 & 30 & 26 \\
37 & 43 & 48 & 44 & 49
\end{array}\right] \\
& G=\left[\begin{array}{ccccc}
5 & 9 & 9 & 6 & 6 \\
10 & 9 & 7 & 9 & 10 \\
8 & 6 & 6 & 9 & 6
\end{array}\right] \text {; } \\
& S^{0}=\left[\begin{array}{lllll}
0 & 4 & 6 & 1 & 0 \\
7 & 6 & 3 & 7 & 6 \\
1 & 5 & 2 & 6 & 0
\end{array}\right] \text {; } \\
& \Psi=\left[\begin{array}{lllll}
1 & 3 & 2 & 1 & 3 \\
1 & 3 & 1 & 2 & 1 \\
3 & 3 & 3 & 3 & 3 \\
3 & 2 & 2 & 3 & 3 \\
2 & 1 & 2 & 1 & 2
\end{array}\right] ; \\
& \Psi_{b}=\left[\begin{array}{lllll}
4 & 5 & 2 & 5 & 4 \\
2 & 2 & 2 & 5 & 3 \\
5 & 4 & 5 & 2 & 3 \\
3 & 2 & 4 & 4 & 2 \\
2 & 1 & 2 & 5 & 2
\end{array}\right] ; \\
& \Omega=\left[\begin{array}{lllll}
2 & 2 & 2 & 1 & 1 \\
2 & 2 & 2 & 2 & 1 \\
2 & 2 & 2 & 1 & 1 \\
1 & 1 & 2 & 1 & 2 \\
2 & 1 & 2 & 2 & 2
\end{array}\right] ; \\
& D=\left[\begin{array}{lllllllllllllllllllllllllllll}
1 & 1 & 2 & 3 & 1 & \vdots & 1 & 3 & 3 & 1 & 1 & \vdots & 3 & 1 & 1 & 1 & 3 & \vdots & 3 & 2 & 1 & 1 & 3 & \vdots & 2 & 2 & 1 & 2 & 3 \\
3 & 3 & 3 & 2 & 1 & \vdots & 2 & 2 & 2 & 2 & 3 & \vdots & 3 & 3 & 3 & 3 & 2 & \vdots & 2 & 1 & 3 & 2 & 2 & \vdots & 2 & 2 & 1 & 2 & 3 \\
1 & 1 & 3 & 3 & 1 & \vdots & 1 & 1 & 3 & 3 & 3 & \vdots & 1 & 3 & 1 & 3 & 1 & \vdots & 2 & 1 & 2 & 1 & 2 & \vdots & 2 & 1 & 3 & 2 & 3 \\
1 & 1 & 1 & 2 & 1 & \vdots & 3 & 3 & 3 & 3 & 3 & \vdots & 3 & 2 & 1 & 1 & 1 & \vdots & 2 & 2 & 2 & 3 & 3 & \vdots & 2 & 2 & 1 & 3 & 2 \\
1 & 2 & 3 & 1 & 2 & \vdots & 2 & 2 & 1 & 3 & 3 & \vdots & 3 & 2 & 2 & 2 & 2 & \vdots & 2 & 3 & 1 & 3 & 1 & \vdots & 3 & 2 & 1 & 2 & 1
\end{array}\right] ;
\end{aligned}
$$




$$
\begin{aligned}
& T_{\mathrm{pr}}=\left[\begin{array}{lllllllllllllllllllllllllllll}
5 & 3 & 5 & 4 & 5 & \vdots & 4 & 5 & 4 & 3 & 4 & \vdots & 3 & 4 & 5 & 3 & 4 & \vdots & 4 & 3 & 4 & 4 & 4 & \vdots & 4 & 5 & 5 & 3 & 5 \\
4 & 3 & 5 & 4 & 4 & \vdots & 5 & 5 & 3 & 3 & 3 & \vdots & 4 & 5 & 3 & 3 & 3 & \vdots & 5 & 3 & 4 & 5 & 4 & \vdots & 5 & 3 & 5 & 4 & 5 \\
3 & 5 & 4 & 5 & 4 & \vdots & 4 & 3 & 4 & 5 & 4 & \vdots & 4 & 3 & 3 & 3 & 5 & \vdots & 5 & 5 & 4 & 3 & 3 & \vdots & 5 & 5 & 3 & 4 & 5 \\
3 & 5 & 3 & 5 & 5 & \vdots & 4 & 3 & 4 & 4 & 3 & \vdots & 3 & 4 & 4 & 4 & 3 & \vdots & 3 & 4 & 4 & 4 & 3 & \vdots & 3 & 5 & 3 & 3 & 5 \\
4 & 5 & 3 & 4 & 5 & \vdots & 4 & 4 & 3 & 3 & 5 & \vdots & 5 & 3 & 3 & 4 & 4 & \vdots & 5 & 5 & 4 & 3 & 3 & \vdots & 5 & 3 & 4 & 5 & 5
\end{array}\right] ; \\
& T_{\text {repl }}=\left[\begin{array}{lllll}
9 & 8 & 8 & 8 & 8 \\
7 & 9 & 7 & 9 & 9 \\
7 & 9 & 9 & 9 & 7
\end{array}\right] \\
& B=\left[\begin{array}{llll}
-1 & -1 & -1 & 0
\end{array}\right] ; \\
& C_{\mathrm{UTC}}=\left[\begin{array}{lllll}
5 & 6 & 4 & 7 & 8
\end{array}\right] .
\end{aligned}
$$

The simulator implemented for processing the data either generates them at random or accepts them from the file or the operator of the system introduces them manually. It is possible to alternate data if necessary. In our case the data are obtained from the ready file which was subject to thorough verification [26]. Figure 1 presents the sample way of introducing initial data for the subsequent simulation process.

The data in Figure 1 are subject to the subsequent simulation procedure which generates results shown in Table 1. The main criterion remains the minimal manufacturing time; however, results of the total replacement time, the lost flow capacity, and the remaining capacity are given for comparison reasons. First of all, the strategies of immediate manufacturing and delayed manufacturing are employed; then the algorithm of the maximal flow capacity of the $\alpha$ th production plant $q_{\alpha \_ \text {max }}^{k}$ (max_flow) and the algorithm of the minimal flow capacity of the $\alpha$ th production plant $q_{\alpha \_ \text {min }}^{k}$ (min_flow) are introduced. Finally, the algorithm of the maximal order $q_{z \text { max }}^{k}$ (max_order) and the algorithm of the minimal order $q_{z \_ \text {min }}^{k}$ (min_flow) are introduced. Additionally, results of the 1000 simulations for both manufacturing strategies are presented. The input conditions are the same for simulation experiments in case of the system consisting of either 3 or 4 or 5 manufacturing plants which are subject to the subsequent thorough analysis.

In the case of the three-plant system the total minimal manufacturing time (1997 time units) is obtained by means of carrying out 1000 simulations with the use of the so-called immediate strategy; however, it does not guarantee achieving the lowest value of the total replacement time as well as the lowest value of the lost flow capacity and the highest value of the remaining capacity.

In the case of the four-plant system the total minimal manufacturing time (1509 time units) is also obtained by means of carrying out 1000 simulations with the use of the so-called immediate strategy. This time, it achieves the lowest value of the total replacement time as well as the lowest value of the lost flow capacity. Unfortunately, it delivers the worst value of the remaining capacity.

In the case of the five-plant system the total minimal manufacturing time (1242 time units) is again obtained by means of carrying out 1000 simulations with the use of the immediate strategy. It also reaches the lowest value of the total replacement time as well as the lowest value of the lost flow capacity. Again, it delivers the worst value of the remaining capacity. In conclusion, all best results are obtained by means of the immediate manufacturing strategy.

Let us assume that the results shown in Table 2 enable us to take the total minimal manufacturing times for granted in each manufacturing system, that is, consisting of 3,4 , and 5 available plants on the condition that they are to be used simultaneously. The total minimal manufacturing time in each system consisting of $A$ subsystems is the longest time in the $\alpha$ th subsystem belonging to this system according to the table of results.

The times shown in Table 2 represent sequences of making orders in each $\alpha$ th plant. The sequences were obtained by means of simulation processes carried out for the data of this case study. Each result of the simulation process generates the best sequence of orders made in the separate plants of each system. For illustration reasons Table 3 shows time scheduling in case of implementing the time minimisation criterion for the 5-plant system. It forms the basis for further analysis.

The sequences for minimising the total manufacturing time are given in Table 4 and, at the same time, they are abbreviated to the adequate shortcuts where $\rho_{A}^{\beta}$ is the $\beta$ th sequence in the system consisting of $A$ identical plants (it must be emphasised that for the needs of the case study the initial state is the same for each $\alpha$ th subsystem).

The basis for improving the sample time scheduling approach is the minimal total manufacturing time obtained 


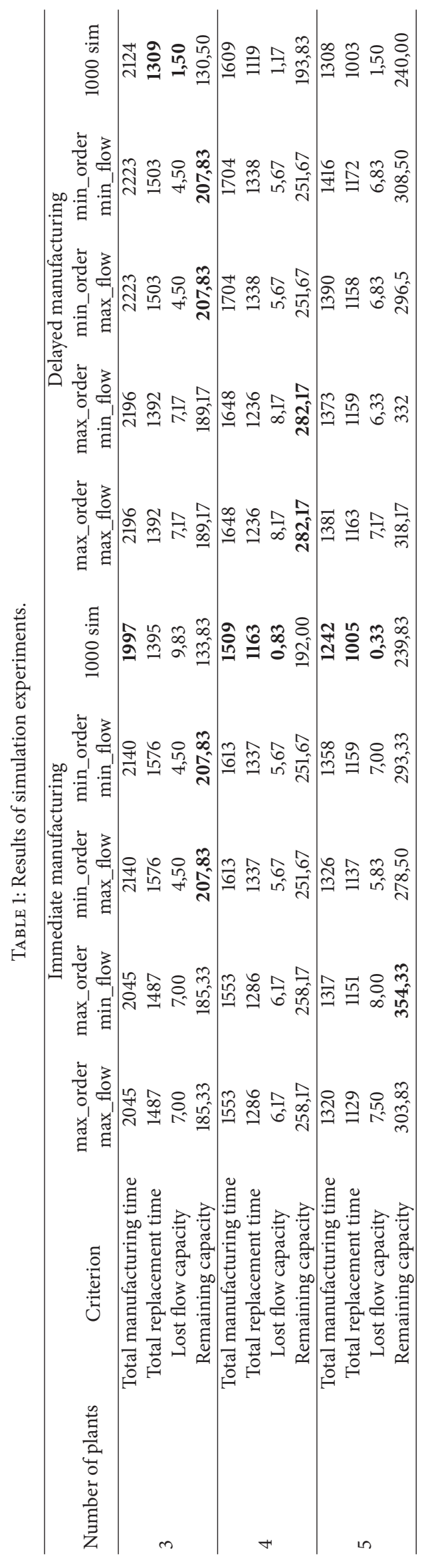




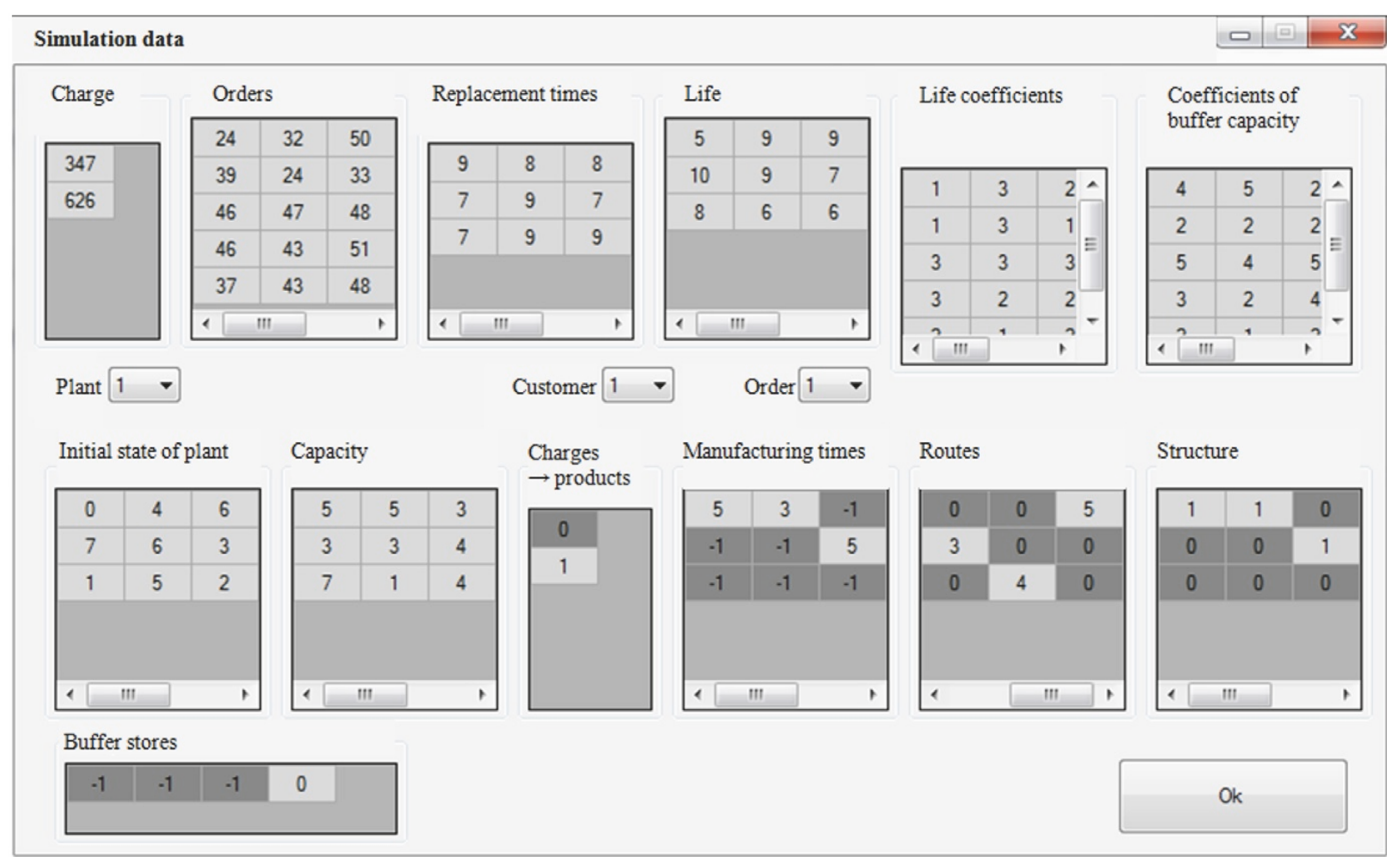

FIGURE 1: Sample initial data introduction for $\alpha=1, m=1$, and $n=1$.

TABLE 2: Manufacturing times in autonomous systems.

\begin{tabular}{lccc}
\hline$\alpha$ & \multicolumn{3}{c}{ System } \\
& 3 plants & 4 plants & 5 plants \\
\hline 1 & 1968 & 1466 & $\mathbf{1 2 4 2}$ \\
2 & $\mathbf{1 9 9 7}$ & 1489 & $\mathbf{1 2 4 2}$ \\
3 & 1957 & 1498 & 1196 \\
4 & $\times$ & $\mathbf{1 5 0 9}$ & 1235 \\
5 & $\times$ & $\times$ & 1168 \\
\hline Total manufacturing time in the system & $\mathbf{1 9 9 7}$ & $\mathbf{1 5 0 9}$ & $\mathbf{1 2 4 2}$ \\
\hline
\end{tabular}

by means of implementing the immediate manufacturing strategy: 1997 for the 3-plant system, 1509 for the 4-plant system, and 1242 for the 5-plant system (see Table 1). The results for the 5-plant system are shown graphically as a result of the simulation process (Figure 2).

The simulation results are associated with system plants; however, it is possible to move the manufacturing process to any other plant in the system as they perform the same production operations. Following this kind of reasoning it was assumed that it is possible to transfer the sequence of orders adjusted to a certain plant on the basis of the simulation results to any other plant in the discussed system. To carry out this process it is necessary to implement permutation without repetitions in order to find the solution which can minimise manufacturing costs to meet the criterion of minimising costs. It became possible only by comparing the results obtained by the method using the permutations without repetitions. In case of 3 plants there are $3 !=6$ possible solutions (see Table 5), 4 plants generate $4 !=24$
TABLE 3: Time scheduling in case of implementing the time minimisation criterion.

\begin{tabular}{lccccc}
\hline Order number & $z_{1,1}^{0}$ & $z_{1,2}^{0}$ & $z_{1,3}^{0}$ & $z_{1,4}^{0}$ & $z_{1,5}^{0}$ \\
Plant number & 1 & 5 & 3 & 4 & 4 \\
Start & 119 & 442 & 0 & 813 & 0 \\
End & 353 & 640 & 363 & 1235 & 161 \\
\hline Order number & $z_{2,1}^{0}$ & $z_{2,2}^{0}$ & $z_{2,3}^{0}$ & $z_{2,4}^{0}$ & $z_{2,5}^{0}$ \\
Plant number & 2 & 5 & 2 & 1 & 4 \\
Start & 900 & 0 & 624 & 540 & 462 \\
End & 1242 & 134 & 917 & 722 & 698 \\
\hline Order number & $z_{3,1}^{0}$ & $z_{3,2}^{0}$ & $z_{3,3}^{0}$ & $z_{3,4}^{0}$ & $z_{3,5}^{0}$ \\
Plant number & 3 & 5 & 1 & 3 & 1 \\
Start & 733 & 901 & 708 & 344 & 336 \\
End & 996 & 1168 & 970 & 503 & 554 \\
\hline Order number & $z_{4,1}^{0}$ & $z_{4,2}^{0}$ & $z_{4,3}^{0}$ & $z_{4,4}^{0}$ & $z_{4,5}^{0}$ \\
Plant number & 3 & 5 & 2 & 1 & 4 \\
Start & 979 & 622 & 0 & 0 & 675 \\
End & 1196 & 922 & 334 & 134 & 828 \\
\hline Order number & $z_{5,1}^{0}$ & $z_{5,2}^{0}$ & $z_{5,3}^{0}$ & $z_{5,4}^{0}$ & $z_{5,5}^{0}$ \\
Plant number & 3 & 2 & 4 & 1 & 5 \\
Start & 488 & 319 & 144 & 909 & 120 \\
End & 751 & 650 & 479 & 1242 & 469 \\
\hline
\end{tabular}

possible solutions (see Table 6), and, finally, 5 plants deliver $5 !=120$ possible solutions (see Table 7).

In the 3-plant system total manufacturing costs are minimised in the case of the combination of production 
TABLE 4: Sequences leading to minimisation the total manufacturing time.

\begin{tabular}{|c|c|c|}
\hline Number of plant & Sequence in the 3-plant system & Production time \\
\hline 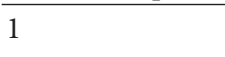 & $\rho_{3}^{1}=z_{5,1}^{k} z_{3,3}^{k} z_{1,3}^{k} z_{5,4}^{k} z_{4,4}^{k} z_{2,4}^{k} z_{3,5}^{k} z_{1,5}^{k} z_{2,2}^{k}$ & 1968 \\
\hline 2 & $\rho_{3}^{2}=z_{4,5}^{k} z_{1,4}^{k} z_{1,2}^{k} z_{5,3}^{k} z_{4,1}^{k} z_{3,2}^{k} z_{4,2}^{k} z_{2,1}^{k}$ & 1997 \\
\hline 3 & $\rho_{3}^{3}=z_{5,2}^{k} z_{3,1}^{k} z_{2,5}^{k} z_{4,3}^{k} z_{5,5}^{k} z_{1,1}^{k} z_{2,3}^{k} z_{3,4}^{k}$ & 1957 \\
\hline Number of plant & Sequence in the 4-plant system & Production time \\
\hline 1 & $\rho_{4}^{1}=z_{2,2}^{k} z_{4,1}^{k} z_{1,2}^{k} z_{3,4}^{k} z_{1,1}^{k} z_{1,5}^{k} z_{4,4}^{k} z_{2,3}^{k}$ & 1466 \\
\hline 2 & $\rho_{4}^{2}=z_{3,2}^{k} z_{2,}^{k}$ & 1489 \\
\hline 3 & $\rho_{4}^{3}=z_{3,3}^{k} z_{1,4}^{k} z_{3,1}^{k} z_{5,2}^{k} z_{5,1}^{k} z_{3,5}^{k}$ & 1498 \\
\hline 4 & $\rho_{4}^{4}=z_{5,5}^{k} z_{5,3}^{k} z_{2,5}^{k} z_{2,1}^{k} z_{1,3}^{k}$ & 1509 \\
\hline Number of plant & Sequence in the 5-plant system & Production time \\
\hline 1 & $\rho_{5}^{1}=z_{4,4}^{k} z_{1,1}^{k} z_{3,5}^{k} z_{2,4}^{k} z_{3,3}^{k} z_{5,4}^{k}$ & 1242 \\
\hline 2 & $\rho_{5}^{2}=z_{4,3}^{k} z_{5,2}^{k} z_{2,3}^{k} z_{2,1}^{k}$ & 1242 \\
\hline 3 & $\rho_{5}^{3}=z_{1,3}^{k} z_{3,4}^{k} z_{5,1}^{k} z_{3,1}^{k} z_{4,1}^{k}$ & 1196 \\
\hline 4 & $\rho_{5}^{4}=z_{1,5}^{k} z_{5,3}^{k} z_{2,5}^{k} z_{4,5}^{k} z_{1,4}^{k}$ & 1235 \\
\hline 5 & $\rho_{5}^{5}=z_{2,2}^{k} z_{5,5}^{k} z_{1,2}^{k} z_{4,2}^{k} z_{3,2}^{k}$ & 1168 \\
\hline
\end{tabular}

TABLE 5: Manufacturing costs in the three-plant system (all data).

\begin{tabular}{|c|c|c|c|c|c|c|c|c|c|c|c|c|c|}
\hline \multirow{2}{*}{ Plant } & \multirow{2}{*}{ Unit cost } & \multicolumn{12}{|c|}{ Combination number of production sequences } \\
\hline & & \multicolumn{2}{|c|}{1} & \multicolumn{2}{|c|}{2} & \multicolumn{2}{|c|}{3} & \multicolumn{2}{|c|}{4} & \multicolumn{2}{|c|}{5} & \multicolumn{2}{|c|}{6} \\
\hline 1 & 5 & $\rho_{3}^{1}$ & 9840 & $\rho_{3}^{1}$ & 9840 & $\rho_{3}^{2}$ & 9985 & $\rho_{3}^{2}$ & 9985 & $\rho_{3}^{3}$ & 9785 & $\rho_{3}^{3}$ & 9785 \\
\hline 2 & 6 & $\rho_{3}^{2}$ & 11982 & $\rho_{3}^{3}$ & 11742 & $\rho_{3}^{1}$ & 11808 & $\rho_{3}^{3}$ & 11742 & $\rho_{3}^{1}$ & 11808 & $\rho_{3}^{2}$ & 11982 \\
\hline 3 & 4 & $\rho_{3}^{3}$ & 7828 & $\rho_{3}^{2}$ & 7988 & $\rho_{3}^{3}$ & 7828 & $\rho_{3}^{1}$ & 7872 & $\rho_{3}^{2}$ & 7988 & $\rho_{3}^{1}$ & 7872 \\
\hline \multicolumn{2}{|c|}{ Total costs } & \multicolumn{2}{|c|}{29650} & \multicolumn{2}{|c|}{29570} & \multicolumn{2}{|c|}{29621} & \multicolumn{2}{|c|}{29599} & \multicolumn{2}{|c|}{29581} & \multicolumn{2}{|c|}{29639} \\
\hline
\end{tabular}

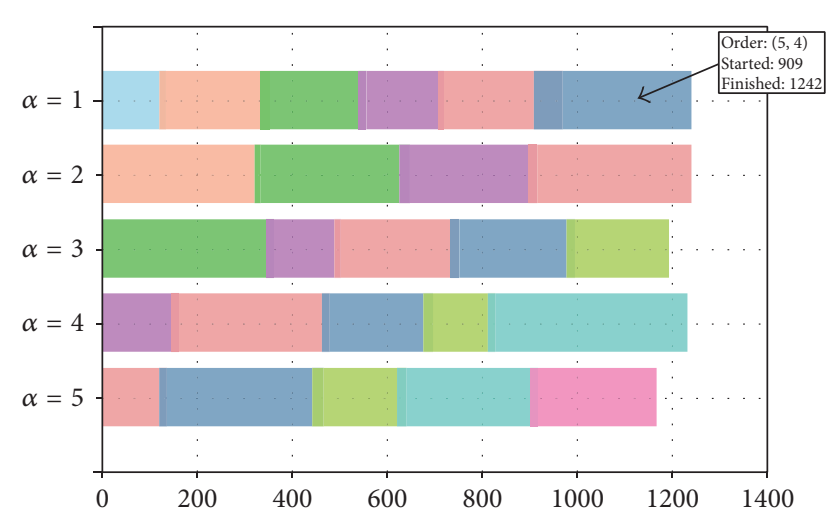

Figure 2: Results of the simulation process for $A=5$.

sequence number 2 by 80 monetary units comparing them with the best result for 1000 simulations (see Table 1).

In the 4-plant system total manufacturing costs are minimised in the case of the combination of production sequence number 17 by 97 monetary units comparing them with the best result for 1000 simulations (see Table 1).

On the basis of comparison results shown in Table 7, the course of the manufacturing process in the five-plant system was illustrated. Total manufacturing costs are minimised in the case of the combinations of production sequence numbers 15 and 33 which are the best in terms of seeking the minimal costs of making orders. As seen in Table 7, implementing these two combinations resulting from searching for the solution to minimise the total manufacturing costs lowers them by 131 monetary units comparing them with the best result for 1000 simulations (see Table 1). Finding the time minimising combinations numbers 15 and 33 enables us to adjust the best sequence of orders to the plants which minimise the summarised manufacturing time shown in Table 8.

The results obtained on the basis of the data implemented for the purpose of the case study let us come to a conclusion that under the very theoretical conditions specified in the article it can be expected that the greater number of manufacturing plants in the system leads to bigger financial savings.

\section{Conclusions}

It can be expected that carrying out more simulation experiments for a certain number of manufacturing plants in the system may deliver even a shorter total manufacturing time which is the time in the system characterised by the longest manufacturing time in its subsystem. This result forms the basis for improving the total manufacturing costs 
TABle 6: Manufacturing costs in the four-plant system (all data).

\begin{tabular}{|c|c|c|c|c|c|c|c|c|c|c|c|c|c|}
\hline \multirow{3}{*}{$\begin{array}{l}\text { Plant } \\
1\end{array}$} & \multirow{3}{*}{$\begin{array}{c}\text { Unit cost } \\
5\end{array}$} & \multicolumn{12}{|c|}{ Combination number of production sequences } \\
\hline & & \multicolumn{2}{|c|}{1} & \multicolumn{2}{|c|}{2} & \multicolumn{2}{|c|}{3} & \multicolumn{2}{|c|}{4} & \multicolumn{2}{|c|}{5} & \multicolumn{2}{|c|}{6} \\
\hline & & $\rho_{4}^{1}$ & 7330 & $\rho_{4}^{1}$ & 7330 & $\rho_{4}^{1}$ & 7330 & $\rho_{4}^{1}$ & 7330 & $\rho_{4}^{1}$ & 7330 & $\rho_{4}^{1}$ & 7330 \\
\hline 2 & 6 & $\rho_{4}^{2}$ & 8934 & $\rho_{4}^{2}$ & 8934 & $\rho_{4}^{3}$ & 8988 & $\rho_{4}^{3}$ & 8988 & $\rho_{4}^{4}$ & 9054 & $\rho_{4}^{4}$ & 9054 \\
\hline 3 & 4 & $\rho_{4}^{3}$ & 5992 & $\rho_{4}^{4}$ & 6036 & $\rho_{4}^{4}$ & 6036 & $\rho_{4}^{2}$ & 5956 & $\rho_{4}^{2}$ & 5956 & $\rho_{4}^{3}$ & 5992 \\
\hline 4 & 7 & $\rho_{4}^{4}$ & 10563 & $\rho_{4}^{3}$ & 10486 & $\rho_{4}^{2}$ & 10423 & $\rho_{4}^{4}$ & 10563 & $\rho_{4}^{3}$ & 10486 & $\rho_{4}^{2}$ & 10423 \\
\hline \multicolumn{2}{|c|}{ Total costs } & \multicolumn{2}{|c|}{32819} & \multicolumn{2}{|c|}{32786} & \multicolumn{2}{|c|}{32777} & \multicolumn{2}{|c|}{32837} & \multicolumn{2}{|c|}{32826} & \multicolumn{2}{|c|}{32799} \\
\hline \multirow{2}{*}{ Plant } & \multirow{2}{*}{ Unit cost } & \multicolumn{12}{|c|}{ Combination number of production sequences } \\
\hline & & \multicolumn{2}{|c|}{7} & \multicolumn{2}{|c|}{8} & & & \multicolumn{2}{|c|}{10} & \multicolumn{2}{|c|}{11} & \multicolumn{2}{|c|}{12} \\
\hline 1 & 5 & $\rho_{4}^{2}$ & 7445 & $\rho_{4}^{2}$ & 7445 & $\rho_{4}^{2}$ & 7445 & $\rho_{4}^{2}$ & 7445 & $\rho_{4}^{2}$ & 7445 & $\rho_{4}^{2}$ & 7445 \\
\hline 2 & 6 & $\rho_{4}^{3}$ & 8988 & $\rho_{4}^{3}$ & 8988 & $\rho_{4}^{4}$ & 9054 & $\rho_{4}^{4}$ & 9054 & $\rho_{4}^{1}$ & 8796 & $\rho_{4}^{1}$ & 8796 \\
\hline 3 & 4 & $\rho_{4}^{4}$ & 6036 & $\rho_{4}^{1}$ & 5864 & $\rho_{4}^{1}$ & 5864 & $\rho_{4}^{3}$ & 5992 & $\rho_{4}^{3}$ & 5992 & $\rho_{4}^{4}$ & 6036 \\
\hline 4 & 7 & $\rho_{4}^{1}$ & 10262 & $\rho_{4}^{4}$ & 10563 & $\rho_{4}^{3}$ & 10486 & $\rho_{4}^{1}$ & 10262 & $\rho_{4}^{4}$ & 10563 & $\rho_{4}^{3}$ & 10486 \\
\hline \multicolumn{2}{|c|}{ Total costs } & \multicolumn{2}{|c|}{32731} & \multicolumn{2}{|c|}{32860} & \multicolumn{2}{|c|}{32849} & & & & 96 & & 763 \\
\hline Plant & Unit cost & & & & & binat & number & nrod & on sequ & & & & \\
\hline & & & & & 4 & & & & & & & & 8 \\
\hline 1 & 5 & $\rho_{4}^{3}$ & 7490 & $\rho_{4}^{3}$ & 7490 & $\rho_{4}^{3}$ & 7490 & $\rho_{4}^{3}$ & 7490 & $\rho_{4}^{3}$ & 7490 & $\rho_{4}^{3}$ & 7490 \\
\hline 2 & 6 & $\rho_{4}^{4}$ & 9054 & $\rho_{4}^{4}$ & 9054 & $\rho_{4}^{1}$ & 8796 & $\rho_{4}^{1}$ & 8796 & $\rho_{4}^{2}$ & 8934 & $\rho_{4}^{2}$ & 8934 \\
\hline 3 & 4 & $\rho_{4}^{1}$ & 5864 & $\rho_{4}^{2}$ & 5956 & $\rho_{4}^{2}$ & 5956 & $\rho_{4}^{4}$ & 6036 & $\rho_{4}^{4}$ & 6036 & $\rho_{4}^{1}$ & 5864 \\
\hline 4 & 7 & $\rho_{4}^{2}$ & 10423 & $\rho_{4}^{1}$ & 10262 & $\rho_{4}^{4}$ & 10563 & $\rho_{4}^{2}$ & 10423 & $\rho_{4}^{1}$ & 10262 & $\rho_{4}^{4}$ & 10563 \\
\hline Tota & osts & & & & & & & & & & 22 & & 851 \\
\hline & Unit cost & & & & & binat & number & piod & on sequ & & & & \\
\hline & Uitit cost & & & & & & & & & & 3 & & 4 \\
\hline 1 & 5 & $\rho_{4}^{4}$ & 7545 & $\rho_{4}^{4}$ & 7545 & $\rho_{4}^{4}$ & 7545 & $\rho_{4}^{4}$ & 7545 & $\rho_{4}^{4}$ & 7545 & $\rho_{4}^{4}$ & 7545 \\
\hline 2 & 6 & $\rho_{4}^{1}$ & 8796 & $\rho_{4}^{1}$ & 8796 & $\rho_{4}^{2}$ & 8934 & $\rho_{4}^{2}$ & 8934 & $\rho_{4}^{3}$ & 8988 & $\rho_{4}^{3}$ & 8988 \\
\hline 3 & 4 & $\rho_{4}^{2}$ & 5956 & $\rho_{4}^{3}$ & 5992 & $\rho_{4}^{3}$ & 5992 & $\rho_{4}^{1}$ & 5864 & $\rho_{4}^{1}$ & 5864 & $\rho_{4}^{2}$ & 5956 \\
\hline 4 & 7 & $\rho_{4}^{3}$ & 10486 & $\rho_{4}^{2}$ & 10423 & $\rho_{4}^{1}$ & 10262 & $\rho_{4}^{3}$ & 10486 & $\rho_{4}^{2}$ & 10423 & $\rho_{4}^{1}$ & 10262 \\
\hline Tota & costs & & & & 56 & & 33 & & & & 20 & & 751 \\
\hline
\end{tabular}

TABLE 7: Manufacturing costs in the five-plant system (chosen data).

\begin{tabular}{|c|c|c|c|c|c|c|c|c|c|c|c|c|c|}
\hline \multirow{3}{*}{$\begin{array}{l}\text { Plant } \\
1\end{array}$} & \multirow{3}{*}{$\begin{array}{c}\text { Unit cost } \\
5\end{array}$} & \multicolumn{12}{|c|}{ Combination number of production sequences } \\
\hline & & \multicolumn{2}{|c|}{11} & \multicolumn{2}{|c|}{12} & \multicolumn{2}{|c|}{13} & \multicolumn{2}{|c|}{14} & \multicolumn{2}{|c|}{15} & \multicolumn{2}{|c|}{16} \\
\hline & & $\rho_{5}^{1}$ & 6210 & $\rho_{5}^{1}$ & 6210 & $\rho_{5}^{1}$ & 6210 & $\rho_{5}^{1}$ & 6210 & $\rho_{5}^{1}$ & 6210 & $\rho_{5}^{1}$ & 6210 \\
\hline 2 & 6 & $\rho_{5}^{3}$ & 7176 & $\rho_{5}^{3}$ & 7176 & $\rho_{5}^{4}$ & 7410 & $\rho_{5}^{4}$ & 7410 & $\rho_{5}^{4}$ & 7410 & $\rho_{5}^{4}$ & 7410 \\
\hline 3 & 4 & $\rho_{5}^{2}$ & 4968 & $\rho_{5}^{2}$ & 4968 & $\rho_{5}^{5}$ & 4672 & $\rho_{5}^{5}$ & 4672 & $\rho_{5}^{2}$ & 4968 & $\rho_{5}^{2}$ & 4968 \\
\hline 4 & 7 & $\rho_{5}^{4}$ & 8645 & $\rho_{5}^{5}$ & 8176 & $\rho_{5}^{2}$ & 8694 & $\rho_{5}^{3}$ & 8372 & $\rho_{5}^{3}$ & 8372 & $\rho_{5}^{5}$ & 8176 \\
\hline 5 & 8 & $\rho_{5}^{5}$ & 9344 & $\rho_{5}^{4}$ & 9880 & $\rho_{5}^{3}$ & 9568 & $\rho_{5}^{2}$ & 9936 & $\rho_{5}^{5}$ & 9344 & $\rho_{5}^{3}$ & 9568 \\
\hline \multicolumn{2}{|c|}{ Total costs } & \multicolumn{2}{|c|}{36343} & \multicolumn{2}{|c|}{36410} & \multicolumn{2}{|c|}{36554} & \multicolumn{2}{|c|}{36600} & \multicolumn{2}{|c|}{36304} & \multicolumn{2}{|c|}{36332} \\
\hline \multirow{2}{*}{ Plant } & \multirow{2}{*}{ Unit cost } & \multicolumn{12}{|c|}{ Combination number of production sequences } \\
\hline & & \multicolumn{2}{|c|}{17} & \multicolumn{2}{|c|}{18} & \multicolumn{2}{|c|}{19} & \multicolumn{2}{|c|}{20} & \multicolumn{2}{|c|}{21} & \multicolumn{2}{|c|}{33} \\
\hline 1 & 5 & $\rho_{5}^{1}$ & 6210 & $\rho_{5}^{1}$ & 6210 & $\rho_{5}^{1}$ & 6210 & $\rho_{5}^{1}$ & 6210 & $\rho_{5}^{1}$ & 6210 & $\rho_{5}^{2}$ & 6210 \\
\hline 2 & 6 & $\rho_{5}^{4}$ & 7410 & $\rho_{5}^{4}$ & 7410 & $\rho_{5}^{5}$ & 7008 & $\rho_{5}^{5}$ & 7008 & $\rho_{5}^{5}$ & 7008 & $\rho_{5}^{4}$ & 7410 \\
\hline 3 & 4 & $\rho_{5}^{3}$ & 4784 & $\rho_{5}^{3}$ & 4784 & $\rho_{5}^{2}$ & 4968 & $\rho_{5}^{2}$ & 4968 & $\rho_{5}^{3}$ & 4784 & $\rho_{5}^{1}$ & 4968 \\
\hline 4 & 7 & $\rho_{5}^{5}$ & 8176 & $\rho_{5}^{2}$ & 8694 & $\rho_{5}^{3}$ & 8372 & $\rho_{5}^{4}$ & 8645 & $\rho_{5}^{4}$ & 8645 & $\rho_{5}^{3}$ & 8372 \\
\hline 5 & 8 & $\rho_{5}^{2}$ & 9936 & $\rho_{5}^{5}$ & 9344 & $\rho_{5}^{4}$ & 9880 & $\rho_{5}^{3}$ & 9568 & $\rho_{5}^{2}$ & 9936 & $\rho_{5}^{5}$ & 9344 \\
\hline \multicolumn{2}{|c|}{ Total costs } & \multicolumn{2}{|c|}{36516} & \multicolumn{2}{|c|}{36442} & \multicolumn{2}{|c|}{36438} & \multicolumn{2}{|c|}{36399} & \multicolumn{2}{|c|}{36583} & \multicolumn{2}{|c|}{36304} \\
\hline
\end{tabular}


TABLE 8: Manufacturing costs in case of the immediate strategy in the 5-plant system.

\begin{tabular}{|c|c|c|c|c|}
\hline Plant number & Production sequence & End time & Unit cost & Total costs \\
\hline \multicolumn{5}{|c|}{ Best results for 1000 simulations } \\
\hline 1 & $z_{4,4}^{0} z_{1,1}^{0} z_{3,5}^{0} z_{2,4}^{0} z_{3,3}^{0} z_{5,4}^{0}$ & 1242 & 5 & 6210 \\
\hline 2 & $z_{4,3}^{0} z_{5,2}^{0} z_{2,3}^{0} z_{2,1}^{0}$ & 1242 & 6 & 7452 \\
\hline 3 & $z_{1,3}^{0} z_{3,4}^{0} z_{5,1}^{0} z_{3,1}^{0} z_{4,1}^{0}$ & 1196 & 4 & 4784 \\
\hline 4 & $z_{1,5}^{0} z_{5,3}^{0} z_{2,5}^{0} z_{4,5}^{0} z_{1,4}^{0}$ & 1235 & 7 & 8645 \\
\hline 5 & $z_{2,2}^{0} z_{5,5}^{0} z_{1,2}^{0} z_{4,2}^{0} z_{3,2}^{0}$ & 1168 & 8 & 9344 \\
\hline \multicolumn{4}{|c|}{ Total manufacturing costs } & 36435 \\
\hline \multicolumn{5}{|c|}{ Permutations without repetitions, combination 15} \\
\hline 1 & $z_{4,4}^{0} z_{1,1}^{0} z_{3,5}^{0} z_{2,4}^{0} z_{3,3}^{0} z_{5,4}^{0}$ & 1242 & 5 & 6210 \\
\hline 2 & $z_{1,5}^{0} z_{5,3}^{0} z_{2,5}^{0} z_{4,5}^{0} z_{1,4}^{0}$ & 1235 & 6 & 7410 \\
\hline 3 & $z_{4,3}^{0} z_{5,2}^{0} z_{2,3}^{0} z_{2,1}^{0}$ & 1242 & 4 & 4968 \\
\hline 4 & $z_{1,3}^{0} z_{3,4}^{0} z_{5,1}^{0} z_{3,1}^{0} z_{4,1}^{0}$ & 1196 & 7 & 8372 \\
\hline 5 & $z_{2,2}^{0} z_{5,5}^{0} z_{1,2}^{0} z_{4,2}^{0} z_{3,2}^{0}$ & 1168 & 8 & 9344 \\
\hline \multicolumn{4}{|c|}{ Total manufacturing costs } & 36304 \\
\hline \multicolumn{5}{|c|}{ Permutations without repetitions, combination 33} \\
\hline 1 & $z_{4,3}^{0} z_{5,2}^{0} z_{2,3}^{0} z_{2,1}^{0}$ & 1242 & 5 & 6210 \\
\hline 2 & 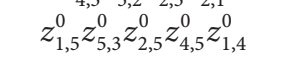 & 1235 & 6 & 7410 \\
\hline 3 & $z_{4,4}^{0} z_{1,1}^{0} z_{3,5}^{0} z_{2,4}^{0} z_{3,3}^{0} z_{5,4}^{0}$ & 1242 & 4 & 4968 \\
\hline 4 & $z_{1,3}^{0} z_{3,4}^{0} z_{5,1}^{0} z_{3,1}^{0} z_{4,1}^{0}$ & 1196 & 7 & 8372 \\
\hline 5 & $z_{2,2}^{0} z_{5,5}^{0} z_{1,2}^{0} z_{4,2}^{0} z_{3,2}^{0}$ & 1168 & 8 & 9344 \\
\hline \multicolumn{4}{|c|}{ Total manufacturing costs } & 36304 \\
\hline
\end{tabular}

by means of seeking for such a combination of adjusting the sequence of production to plants which minimise the total manufacturing costs. It also seems reasonable to implement other criteria, that is, either the criterion of minimising lost capacity or the criterion of maximising the remaining capacity or the criterion of minimising the replacement time. Nevertheless, the assumptions for the mentioned criteria differ from the ones presented in the case study, emphasising both the criterion of minimising the manufacturing time and the criterion of minimising manufacturing costs. Moreover, it is necessary to analyse the behaviour of the system in case of various initial states obtained at random from data characterised by the same range for drawing.

\section{Symbols}

$\alpha$ : The number of the subsystem, $\alpha=1, \ldots, A$

$b_{\alpha / j}^{k}$ : The state of the $j$ th buffer store in the $\alpha$ th manufacturing plant at the $k$ th stage

$b_{j}^{\alpha}$ : The base capacity of the buffer store placed behind the $j$ th tool stand in the $\alpha$ th manufacturing system

$c_{\alpha}$ : The cost of one unit time of operating the $\alpha$ th plant

$C_{\text {UTC }}$ : The vector of unit time costs

$B: \quad$ The structure vector of base capacity of buffer stores

$B^{k}: \quad$ The state of the buffer store $d_{j, n}: \quad$ The number of the $i$ th tool in the $j$ th work stand used for making the $n$th type product

$D: \quad$ The route matrix

$e_{i, j}^{\alpha}$ : The $i$ th tool in the $j$ th work stand in the $\alpha$ th manufacturing plant

E: $\quad$ The matrix of structure

$g_{i, j}: \quad$ The number of base elements of a product which can be made by the $i$ th tool in the $j$ th work stand before it becomes worn out

G: $\quad$ The base life matrix

$k$ : $\quad$ The stage number resulting from the preceding decision in the system, $k=1, \ldots, K$

$m: \quad$ The number of the customer, $m=1, \ldots, M$

$n: \quad$ The type of order, $n=1, \ldots, N$

$p(\alpha)_{i, j}^{k}$ : The base capacity of the $i$ th tool in the $j$ th stand in the $\alpha$ th manufacturing system at the $k$ th stage

$P^{k}: \quad$ The base matrix of flow capacity

$P_{\alpha}^{k}: \quad$ Matrix of flow capacity

$Q_{C}: \quad$ The criterion of minimising manufacturing costs

$Q_{T}$ : The criterion of minimising the manufacturing time

$q_{\alpha \text { max }}^{k}$ : The algorithm of the maximal flow capacity of the $\alpha$ th production plant 


\begin{tabular}{|c|c|}
\hline$q_{\alpha \_ \text {min }}^{k}:$ & $\begin{array}{l}\text { The algorithm of the minimal flow } \\
\text { capacity of the } \alpha \text { th production plant }\end{array}$ \\
\hline$q_{z \_\max }^{k}:$ & The algorithm of the maximal order \\
\hline$q_{z \_\min }:$ & The algorithm of the minimal order \\
\hline$s(\alpha)_{i, j}^{k}:$ & $\begin{array}{l}\text { The base state of the } i \text { th tool in the } j \text { th } \\
\text { stand in the } \alpha \text { th manufacturing system } \\
\text { at the } k \text { th stage }\end{array}$ \\
\hline$s(\alpha)_{(m, n) /(i, j)}^{k}$ & $\begin{array}{l}\text { The state of the } i \text { th tool in the } j \text { th stand } \\
\text { in case of making the } n \text {th product for } \\
\text { the } m \text { th customer }\end{array}$ \\
\hline$S^{k}:$ & The $k$ th state of the system \\
\hline$S_{\alpha}^{k}:$ & $\begin{array}{l}\text { The } k \text { th state of the } \alpha \text { th system } \\
\text { manufacturing subsystem }\end{array}$ \\
\hline$x_{m, n}^{k}:$ & $\begin{array}{l}\text { The number of units of the } n \text {th product } \\
\text { for the } m \text { th customer made at the } k \text { th } \\
\text { stage }\end{array}$ \\
\hline$x(\alpha)_{(m, n) /(i, j)}^{k}:$ & $\begin{array}{l}\text { The amount of the } n \text {th product for the } \\
m \text { th customer made by the } i \text { th tool in } \\
\text { the } j \text { th stand in the } \alpha \text { th manufacturing } \\
\text { system at the } k \text { th stage }\end{array}$ \\
\hline$z_{m, n}^{k}:$ & $\begin{array}{l}\text { The } n \text {th order of the } m \text { th customer at } \\
\text { the } k \text { th stage }\end{array}$ \\
\hline$Z^{k}:$ & The matrix of orders in the $k$ th stage \\
\hline$\tau_{(m, n) /(i, j)}^{\mathrm{pr}}$ & $\begin{array}{l}\text { The manufacturing time of one unit of } \\
\text { the } n \text {th product for the } m \text { th customer } \\
\text { by the } i \text { th tool of the } j \text { th work stand }\end{array}$ \\
\hline$\tau_{j}^{\mathrm{repl}}:$ & $\begin{array}{l}\text { The replacement time of the } j \text { th work } \\
\text { stand }\end{array}$ \\
\hline$T^{\mathrm{pr}}:$ & The matrix of manufacturing times \\
\hline$T^{\text {repl }}:$ & The vector of replacement times \\
\hline$\xi_{\alpha}^{k}:$ & $\begin{array}{l}\text { The coefficient of flow capacity of the } \\
\text { production plant }\end{array}$ \\
\hline$\Psi_{b}:$ & $\begin{array}{l}\text { The matrix of coefficients of the buffer } \\
\text { stores }\end{array}$ \\
\hline$\psi_{j, n}^{\alpha}:$ & $\begin{array}{l}\text { The coefficient of the } j \text { th buffer store in } \\
\text { case of storing the } n \text {th type product in } \\
\text { the } \alpha \text { th manufacturing system }\end{array}$ \\
\hline$\psi_{(m, n) /(i, j)}:$ & $\begin{array}{l}\text { The coefficient determining how many } \\
\text { units of the } n \text {th type order for the } m \text { th } \\
\text { customer can be made by the } i \text { th tool in } \\
\text { the } j \text { th tool stand }\end{array}$ \\
\hline$\Psi:$ & The matrix of coefficients \\
\hline$\omega_{l /(m, n)}:$ & $\begin{array}{l}\text { The adjustment of the } n \text {th order of the } \\
m \text { th customer to the } l \text { th charge }\end{array}$ \\
\hline$\Omega:$ & $\begin{array}{l}\text { The adjustment matrix of charges to } \\
\text { products. }\end{array}$ \\
\hline
\end{tabular}

\section{Conflicts of Interest}

The authors declare that they have no conflicts of interest.

\section{Acknowledgments}

This paper was supported by Project SGS/19/2016 (Silesian University in Opava, School of Business Administration in Karvina): Advanced Mining Methods and Simulation Techniques in the Business Process Domain.

\section{References}

[1] S. Žapčević and P. Butala, "Adaptive process control based on a self-learning mechanism in autonomous manufacturing systems," International Journal of Advanced Manufacturing Technology, vol. 66, no. 9-12, pp. 1725-1743, 2013.

[2] V. Modrak, D. Marton, and S. Bednar, "Comparison of complexity indicators for assessing general process structures," Tehnicki Vjesnik-Technical Gazette, no. 20, pp. 1057-1062, 2013.

[3] V. Modrak, D. Marton, and S. Bednar, "Modeling and determining product variety for mass-customized manufacturing," in Proceedings of the CIRP Conference on Assembly Technologies and Systems, CATS 2014, pp. 258-263, deu, May 2014.

[4] W. Chen, D. Huo, W. Xie, X. Teng, and J. Zhang, "Integrated simulation method for interaction between manufacturing process and machine tool," Chinese Journal of Mechanical Engineering (English Edition), vol. 29, no. 6, pp. 1090-1095, 2016.

[5] H.-S. Park and N.-H. Tran, "An autonomous manufacturing system based on swarm of cognitive agents," Journal of Manufacturing Systems, vol. 31, no. 3, pp. 337-348, 2012.

[6] R. S. Omega, V. M. Noel, J. G. Masbad, and L. A. Ocampo, "Modelling supply risks in interdependent manufacturing systems: a case study," Advances in Production Engineering \& Management, vol. 11, no. 2, pp. 115-125, 2016.

[7] M. P. Brundage, Q. Chang, Y. Li, J. Arinez, and G. Xiao, "Sustainable Manufacturing Performance Indicators for a Serial Production Line," IEEE Transactions on Automation Science and Engineering, vol. 13, no. 2, pp. 676-687, 2016.

[8] P. Chhaochhria and S. C. Graves, "A forecast-driven tactical planning model for a serial manufacturing system," International Journal of Production Research, vol. 51, no. 23-24, pp. 6860-6879, 2013.

[9] G. Popovics, A. Pfeiffer, and L. Monostori, "Generic data structure and validation methodology for simulation of manufacturing systems," International Journal of Computer Integrated Manufacturing, vol. 29, no. 12, pp. 1272-1286, 2016.

[10] A. Greasley, "Using system dynamics in a discrete-event simulation study of a manufacturing plant," International Journal of Operations \& Production Management, vol. 25, no. 5-6, pp. 534548, 2005.

[11] R. Malik and R. Leduc, "Hierarchical modelling of manufacturing systems using discrete event systems and the conflict preorder," Discrete Event Dynamic Systems: Theory and Applications, vol. 25, no. 1-2, pp. 177-201, 2015.

[12] Y. Nonaka, Y. Suginishi, A. Lengyel, S. Nagahara, K. Kamoda, and Y. Katsumura, "The S-Model: A digital manufacturing system combined with autonomous statistical analysis and autonomous discrete-event simulation for smart manufacturing," in Proceedings of the 11th IEEE International Conference on Automation Science and Engineering, CASE 2015, pp. 1006-1011, swe, August 2015.

[13] T. Aljuneidi and A. A. Bulgak, "A mathematical model for designing reconfigurable cellular hybrid manufacturingremanufacturing systems," International Journal of Advanced Manufacturing Technology, vol. 87, no. 5-8, pp. 1585-1596, 2016.

[14] B. Bako and P. Božek, "Trends in simulation and planning of manufacturing companies," in Proceedings of the International Conference on Manufacturing Engineering and Materials, ICMEM 2016, pp. 571-575, svk, June 2016.

[15] C. Gershenson and D. Helbing, "When slower is faster," Complexity, vol. 21, no. 2, pp. 9-15, 2015. 
[16] U. Ghani, R. Monfared, and R. Harrison, "Integration approach to virtual-driven discrete event simulation for manufacturing systems," International Journal of Computer Integrated Manufacturing, vol. 28, no. 8, pp. 844-860, 2015.

[17] R. Šperka, "Application of a business economics decisionmaking function in an agent simulation framework," Smart Innovation, Systems and Technologies, vol. 58, pp. 209-218, 2016.

[18] B. Xu, R. Liu, and Z. He, "Individual irrationality, network structure, and collective intelligence: An agent-based simulation approach," Complexity, vol. 21, pp. 44-54, 2016.

[19] X. D. Nie, X. D. Chen, and X. Chen, "Simulation study of flexible manufacturing cell based on token-oriented petri net model," International Journal of Simulation Modelling, vol. 15, no. 3, pp. 566-576, 2016.

[20] J. Du, M. El-Gafy, and D. Zhao, "Optimization of Change Order Management Process with Object-Oriented Discrete Event Simulation: Case Study, Journal of Construction Engineering and Management, vol. 142, no. 4, Article ID 05015018, 2016.

[21] R. Bucki, B. Chramcov, and P. Suchanek, "Heuristic algorithms for manufacturing and replacement strategies of the production system," Journal of Universal Computer Science, vol. 21, no. 4, pp. 503-525, 2015.

[22] H. Fazlollahtabar, M. Saidi-Mehrabad, and J. Balakrishnan, "Mathematical optimization for earliness/tardiness minimization in a multiple automated guided vehicle manufacturing system via integrated heuristic algorithms," Robotics and Autonomous Systems, vol. 72, pp. 131-138, 2015.

[23] P. Schuster, "Optimization of multiple criteria: Pareto efficiency and fast heuristics should be more popular than they are," Complexity, vol. 18, no. 2, pp. 5-7, 2012.

[24] P. Suchánek and R. Bucki, "Business process modeling of logistic production systems," Smart Innovation, Systems and Technologies, vol. 58, pp. 199-207, 2016.

[25] B. Chramcov and R. Bucki, "Logistic modelling of order realization in the complex parallel manufacturing system," in 27th European Conference on Modelling and Simulation (ECMS '13), pp. 657-663, 2013.

[26] B. Chramcov, R. Bucki, and S. Marusza, "Simulation Analysis of the Complex Production System with Interoperation Buffer Stores," Advances in Intelligent Systems and Computing, vol. 210, pp. 423-434, 2013.

[27] R. Ahmadi, "Optimal maintenance scheduling for a complex manufacturing system subject to deterioration," Annals of Operations Research, vol. 217, pp. 1-29, 2014.

[28] M. Freitag and T. Hildebrandt, "Automatic design of scheduling rules for complex manufacturing systems by multi-objective simulation-based optimization," CIRP Annals - Manufacturing Technology, vol. 65, no. 1, pp. 433-436, 2016.

[29] R. Bucki and F. Marecki, Digital Modelling of Discete Processes, Network Integrators Associates, Parkland, Fla, USA, 2006. 


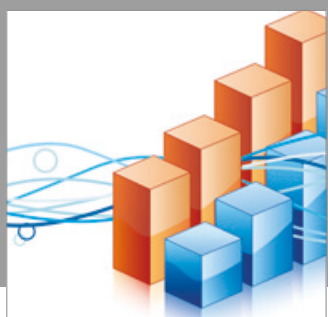

Advances in

Operations Research

vatersals

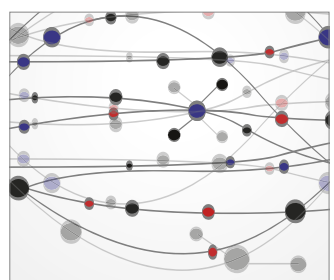

\section{The Scientific} World Journal
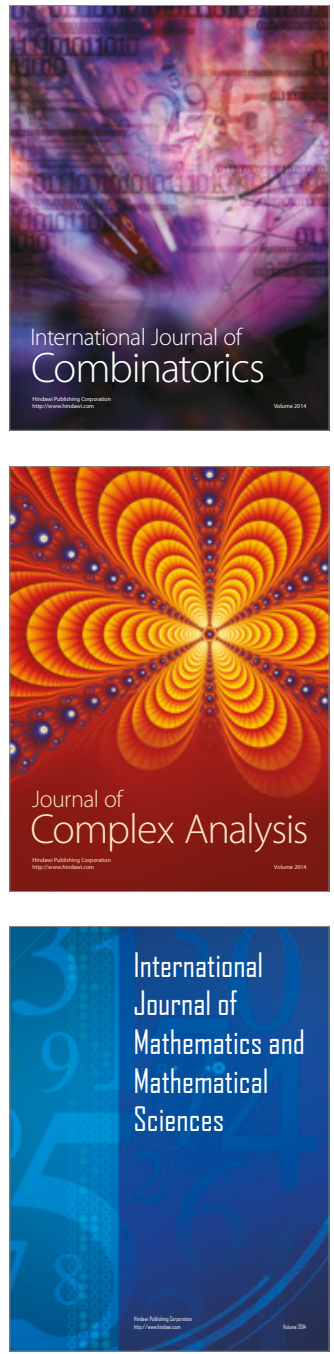
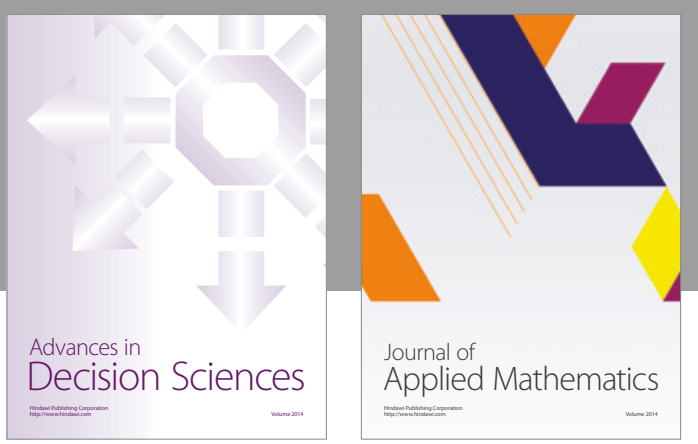

Algebra

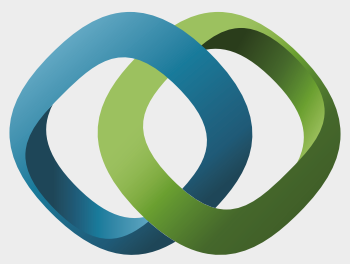

\section{Hindawi}

Submit your manuscripts at

https://www.hindawi.com
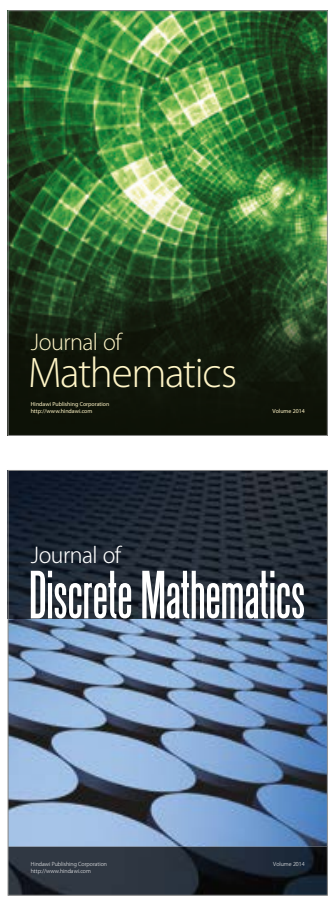

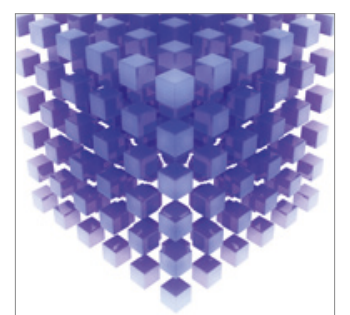

Mathematical Problems in Engineering
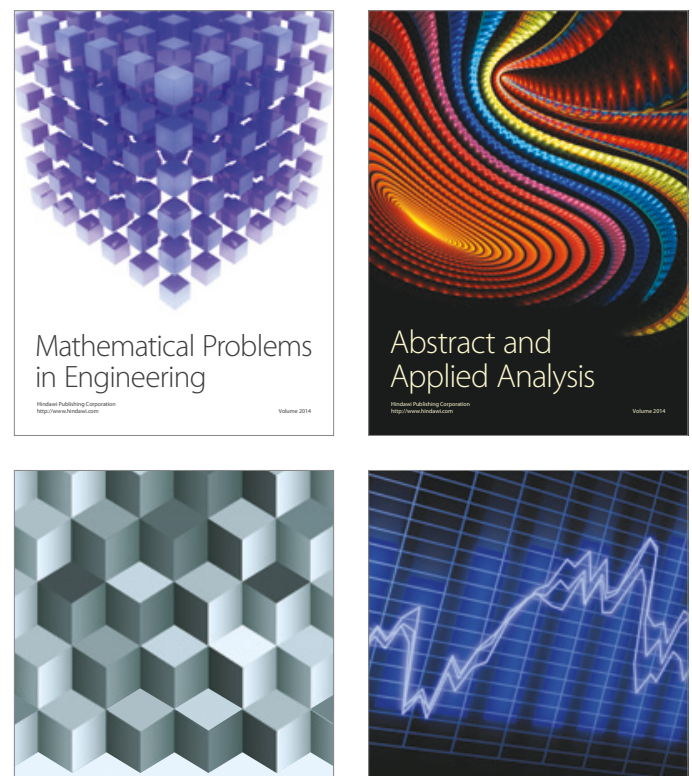

Journal of

Function Spaces

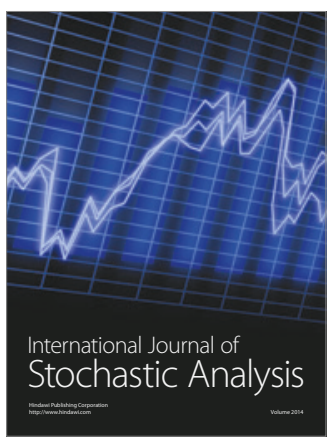

Probability and Statistics
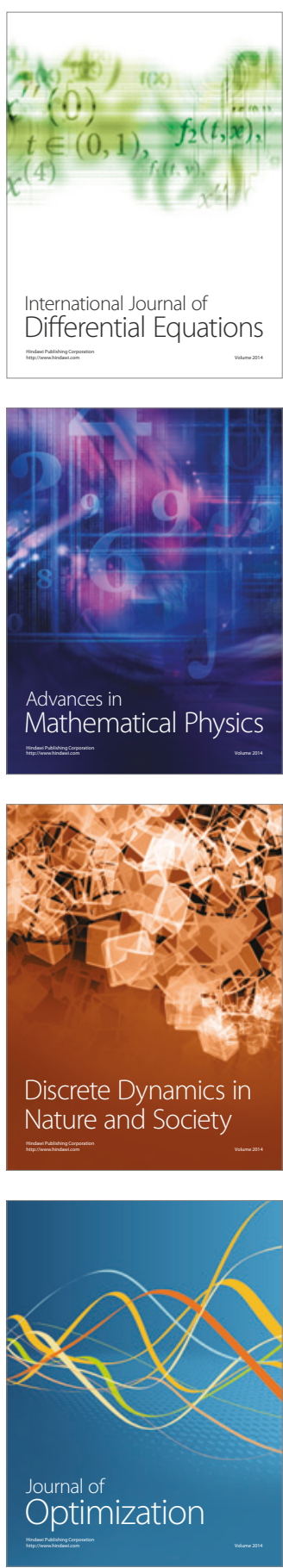